\title{
Hydromagnetic Turbulent Flow Between Two Parallel Infinite Plates
}

\author{
Kennedy John Mwangi Karimi, Dickson Kande Kinyua
}

School of Pure and Applied Sciences, Karatina University, Karatina, Kenya

Email address:

mwanjoken@gmail.com (K. J. M. Karimi), kandesnr@gmail.com (D. K. Kinyua)

To cite this article:

Kennedy John Mwangi Karimi, Dickson Kande Kinyua. Hydromagnetic Turbulent Flow Between Two Parallel Infinite Plates. Science Journal of Applied Mathematics and Statistics. Vol. 5, No. 1, 2017, pp. 31-40. doi: 10.11648/j.sjams.20170501.15

Received: September 28, 2016; Accepted: November 10, 2016; Published: January 21, 2017

\begin{abstract}
In this study we shall investigate hydromagnetic turbulent unsteady flow of an incompressible electrically conducting fluid between two parallel infinite plates. The flow variables such as velocity and thermodynamic properties at every point of fluid vary with respect to time. The effect of an applied transverse magnetic field normal to the main flow direction on the dynamic behavior of the fluid when the lower plate is stationary and the upper plate is impulsively started in opposite direction at constant velocity shall be investigated. Further, we shall investigate how the various parameters such as Peclet Number and Eckert Number affect the flow; in particular, velocity and temperature profiles. A finite difference method shall be used to solve the coupled non-liner and dimensionless partial differential equations governing this problem.
\end{abstract}

Keywords: Magnetohydrodynamics, Incompressible, Dimensionalization, Temperature Profiles

\section{Introduction}

\subsection{The Background of Magnetohydrodynamics}

Magnetohydrodynamics (MHD) is the academic discipline that studies the dynamics of electrically conducting fluids. Examples of such fluids include plasmas, liquid metals, and salt water. The word magnetohydrodynamics (MHD is derived from magneto- meaning magnetic field, and hydromeaning liquid, and dynamics meaning mechanical properties of fluid. Hydrodynamics on the other hand is the study of fluid flow and the forces that cause the flow in the absence of an electromagnetic field. Fluid is considered to be any matter that undergoes deformation when an external force is applied. In MHD, a current is induced when the fluid conductor moves in magnetic field. This is the central point of MHD theory. As a result, when viscous conducting fluid flow in presence of a transverse magnetic field, electromagnetic forces acts on the fluid particles thereby altering their geometry of motion. The momentum equation describing the motion of fluid in MHD includes body forces, which act on fluid particles from a distance. The applied magnetic field acts on both electronic and ionized atoms to produce dynamic effect. This mass motion in turn produces modification in the electromagnetic field. Consequently we have to deal with complicated coupled system of the partial differential equation representing the flow. The mechanical motion of the system can then be described in terms of a single conducting fluid with hydromagnetic variables of density, velocity and pressure. At low frequency it is customary to neglect the displacement current in Ampere's law. This is then the approximation that is called magnetohydrodynamics (Jackson 1975). The flow can either be steady or unsteady, where the flow variables such as velocity and thermodynamics properties are independent of time and dependent on time respectively. Turbulent flow occurs when there are disturbances present in a fluid due to a variety of factors such as porous walls, boundary roughness, and variation in the physical properties of fluid motion among others. Under uniform steady flow, particles of the fluid move in straight path. In the case of turbulent flow, the fluid particles cross each other's paths in disorderly manner varying velocities and pressures. Hartman in (1937) was first to discuss both experimentally and theoretically the hydro magnetic flow between two parallel plates.

\subsection{Boundary Layers}

When a fluid flows over a stationary surface, e.g. the bed of a river or the wall of a pipe, the fluid touching the surface is brought to rest by the shear stress $\tau$ o at the wall. The 
velocity increases from the wall to a maximum in the main stream of the flow, as shown in figure 1 below.

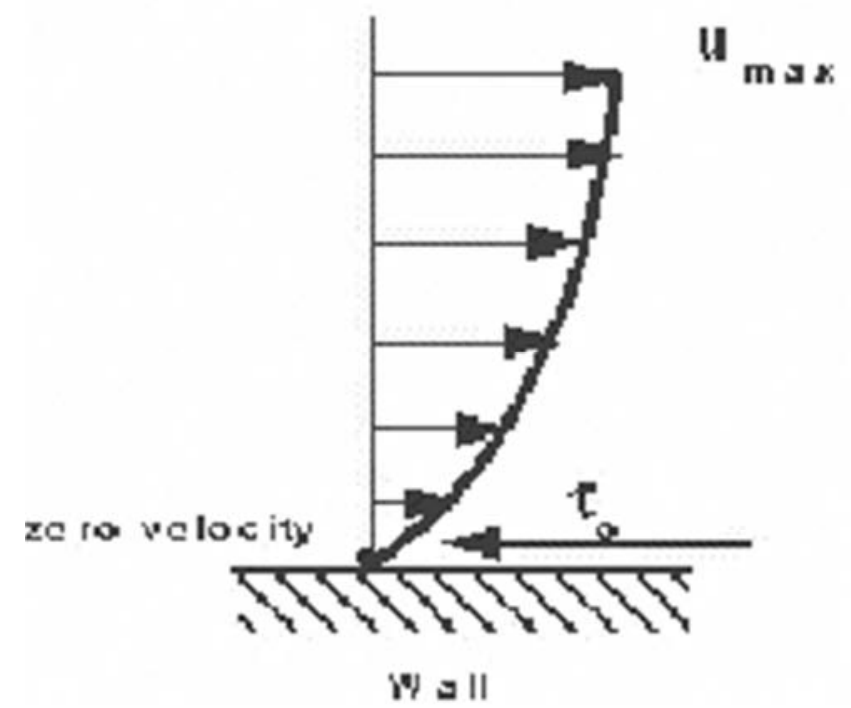

Fig. 1. Boundary layer.

\subsection{Dimensional Analysis}

It is built on the principle of dimensional homogeneity that states that an equation expressing a physical relationship between quantities must be dimensionally homogenous i.e. dimensions of each side of equations must be the same. It affords a means of ascertaining the forms of physical equations from knowledge of relevant variables and their dimensions. It proves a powerful tool in formulating problems that defy analytical solutions and must be solved experimentally.

This method has application in nearly all fields of engineering in particular in fluid mechanics and heat transfer. It is an important tool that presents experimental results in a concise form. In our study, dimensional analysis has been used in the non-dimensionalization of the governing equations. Non-dimensionalization is the partial or full removal of units from an equation involving physical quantities by a suitable substitution of variables. This technique can simplify and parameterize problems where measured units are involved. To non-dimensionalize these equations that describe our flow system appropriately we must do the following:

1) Identify all the independent and dependent variables.

2) Replace each of them with a quantity scaled relative to a characteristic unit of measure to be determined.

3) Divide through by the coefficient of the highest order polynomial or derivative term.

4) Choose judiciously the definition of the characteristic unit for each variable so that the coefficients of as many terms as possible become 1 .

5) Rewrite the system of equations in terms of their new dimensionless quantities.

\subsection{Literature Review}

The first quantitative observations relating to timedependent magnetic and electric fields were made by Faraday (1831) in experiments on the behavior of current in circuits placed in time-varying magnetic fields. In his experiment with mercury as the conducting fluid flowing in a glass tube placed in a magnetic field, he observed that a voltage was induced in a direction perpendicular to both the direction of the flow and magnetic field. From related literature, Riche (1832) discovered that when an electric field is applied to conducting fluid in a direction perpendicular to a magnetic field a force is exerted on the fluid in a direction perpendicular to both the electric field and magnetic field. The observation made by the above scientists' motivated later scholars in electromagnetism leading to extensive research in hydromagnetic flow. As earlier mentioned the flow of conducting fluid between two parallel-insulated plates was first discussed theoretically and experimentally by Hartman(1937) followed by Stokes (1951) who concentrated on the flow of incompressible and viscous fluid past impulsively started infinite flat plates. Stewarson (1951) studied and analyzed the flow of a viscous incompressible fluid past an impulsively started infinite plates and Rossow (1958) researched on the flow of an electrically conducting fluid over a flat plate in the presence of transverse magnetic field. Walker et al (1971) researched on fluid flow in presence of a transverse magnetic field. In the same year Srivastava studied hydromagnetic coutte flow of an electrically conducting, viscous and incompressible fluid in presence of a transverse magnetic field when the plates are non-magnetic and non-conducting with variable suction. Bhaskara and Bathaiah (1980) studied MHD flow of a viscous incompressible and slightly conducting fluid between a parallel flat wall and a wavy wall and evaluated the velocity distribution, the coefficient of skin friction and temperature distribution. Kalyuit et al (1986) studied the development of the flow field of an electrically conducting fluid in an inhomogeneous magnetic field when the induced magnetic field caused by the currents flowing in the liquid is disregarded. They found out that an M-shaped velocity profile develops near the entrance of the magnetic field region where the Lorentz force is not strong enough to divert the flow. Molokov and Allen (1989) studied MHD flow of an incompressible fluid in an open channel in presence of a strong uniform magnetic field while Kumar and Singh (1980) studied unsteady MHD fluid flow through an inclined closed rectangular channel with upper and lower surfaces having varying permeability. In the same year, Ber investigated the motion of an electrically conducting incompressible fluid flowing between two parallel plates in presence of a transverse magnetic field under uniform boundary conditions. Ahmed et al (1992) studied the convective MHD flow past a uniformly moving infinite vertical plate with the magnetic field and the suction applied normal to the plate. Kinyanjui et al (2001) presented their work on MHD free convection heat and mass transfer of a heat generating fluid 
past an impulsively started infinite porous plate with hall current and radiation absorption. Calvert (2002) composed his research article on the fundamental principle of MHD where he showed that the effect of magnetic field on a conducting fluid in motion is to exert a force perpendicular to the magnetic field that tends to make normal velocity equal to the $\mathrm{E} \times \mathrm{B}$ drifts. He asserted that the higher the conductivity, the stronger is this force and the closely is the magnetic field dragged by the fluid (and vice versa) and that the motion of the fluid along the direction of the magnetic field is unaffected. Chandra (2005) studied a steady MHD flow of an electrically conducting fluid between two parallel infinite plates when the upper plate is made to move with constant velocity while the lower plate is stationary. Ramulu et al (2007) studied the effect of hall current on MHD flow and heat transfer along a porous flat plate with mass transfer. He applied numerical methods to obtain the solution. More recently, Samiulhaq et al.(2012) investigated the unsteady MHD flow past an impulsively started vertical plate present in a porous medium with thermal diffusion and ramped wall temperature. Our task will be to investigate MHD flow of an incompressible and viscous conducting fluid between two parallel semi-infinite plates with the upper plate moving with a constant velocity $(-\mathrm{u})$ while the lower plate is stationary in presence of a uniform transverse magnetic field.

\subsection{Statement of the Problem}

In this problem we shall investigate the behavior of a turbulent and unsteady hydromagnetic flow of a viscous conducting fluid between two parallel infinite plates. A uniform magnetic field is applied in a direction normal to the plates. The upper plate is impulsively started in opposite direction to that of the flow parallel to the $\mathrm{x}$-axis at constant velocity $\mathrm{U}_{0}$, while the lower plate is stationery. Our task will be to investigate the velocity profiles and temperature profiles of this turbulent unsteady flow, which has received little attention in previous related research.

\subsection{Justification}

MHD is a field with a wide range of practical applications particularly in engineering. Scientific research in electricity and magnetism is on a worldwide scale. In many engineering practical applications (e.g. an elevator, ball bearing etc) we encounter conducting fluids flowing between moving boundaries. Our problem is a particular case when the upper boundary moves at a constant velocity and in opposite direction to that of flow and the lower boundary is stationery. Most fluids in engineering are unsteady. This wide range of MHD applications in engineering gives our study a practical framework.

\subsection{Objective of the Study}

To investigate;

The effects of Eckert Number on both the temperature profiles and the velocity profile.

\section{Description of the Flow}

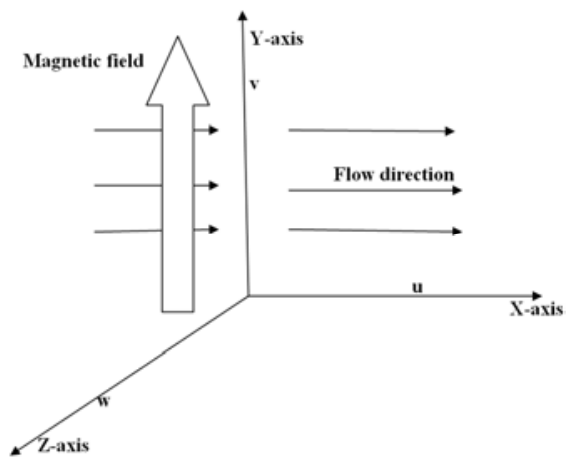

Fig. 2. Magnetic field applied orthogonally to the direction of flow.

We consider the turbulent flow of a viscous electrically conducting incompressible fluid, bounded by an infinite horizontal non porous plate in the presence of a strong magnetic field applied orthogonally to direction of fluid flow. A uniform magnetic field $\mathrm{H}$ is acting at an angle of $90^{\circ}$ to the horizontal plate and the plate is assumed to be electrically non-conducting. Since the plate occupying the plane $y=0$ is of infinite extent, the physical conditions depends on y only.

\subsection{Governing Equations}

The ideal MHD equations consist of the continuity equation, the momentum equation, Amperes law and a temperature evolution equation. In the theory of turbulence, Reynolds decomposition (RD) is an important tool. Reynolds decomposition is a mathematical technique to separate the average and fluctuating parts of a quantity. For example, for a quantity u the decomposition would be

$$
u(x, y, z, t)=\bar{u}(x, y, z, t)+\dot{u}(x, y, z, t)
$$

Where $\bar{u}$ denotes the time average of $u$ (the steady component), and $u$ the fluctuating part (or perturbations). The fluctuating part is defined such that its time average equals zero. This method allows us to simplify the NavierStokes equations by substituting in the sum of the steady component and perturbations to the velocity profile and taking the mean value. This results to a non-linear equation containing a term known as the Reynolds stresses which gives rise to turbulence.

\subsection{Approximation and Assumptions}

1) The fluid is incompressible.

2) Thermo conductivity, electrical conductivity and coefficient of viscosity are constants.

3) There is no external applied electric field $(E=0)$.

4) Compared with the speed of light, the fluid velocity (q) is very small.

5) The fluid does not undergo any chemical reaction.

6) The induced magnetic field produced by the motion of the electrically conducting fluid is negligible and hence magnetic Reynolds number is very small.

7) The plates are insulated 
8) The fluid flow pressure gradient is a constant $\nabla p=\mathrm{P}$

9) The velocity component in the y direction is zero $(v=0)$

\section{Model of the Flow}

\subsection{Equations Governing Fluid Flow}

Conservation of mass The equation of conservation of mass is given by

$$
\frac{\partial \rho}{\partial t}+\nabla \cdot\left(\rho u_{i}\right)=0
$$

Where $\mathrm{i}=1,2,3$

Or

$$
\frac{\partial \rho}{\partial t}+\rho \nabla u_{i}+u_{i} \nabla \rho=0
$$

Assuming that density is a constant this equation reduces to

$$
\nabla u_{i}=0
$$

For a two dimensional flow equation (4) becomes

$$
\frac{\partial u_{1}}{\partial x}+\frac{\partial u_{2}}{\partial y}=0
$$

Or

$$
\frac{\partial u}{\partial x}+\frac{\partial v}{\partial y}=0
$$

For this flow the parallel plates are infinite in length and therefore no flow variable is a function of $\mathrm{x}$. Equation (5) becomes

$$
\frac{\partial v}{\partial y}=0
$$

For turbulent flow we decompose velocity $\mathrm{u}$ into the average component $\bar{u}$ and the fluctuating velocity component $u$ and then take the average

$$
\frac{\partial(\bar{v}+\dot{v})}{\partial y}=0
$$

On averaging we have

$$
\frac{\partial(\overline{\bar{v}+\hat{v}})}{\partial y}=0
$$

or

$$
\frac{\partial \bar{v}}{\partial y}=0
$$

Conservation of momentum

The equation of conservation of momentum is given by

$$
\rho\left[\frac{\partial u}{\partial t}+u \nabla u\right]=-\nabla p+\mu \nabla^{2} u+\mathrm{J} \times \mathrm{B}+\rho \mathrm{g}
$$

This equation is based on the Newton's second law of motion ,that is, the net rate of change of momentum must equal the net sum of forces acting on the fluid. This equation is also known as the Navier-Stokes Equation
The body forces considered in this problem are, $\rho_{e} \mathrm{E}+\mathrm{J} \times$ $\mathrm{B}$ and $\rho \mathrm{g}$, that is, the electromagnetic force and gravity respectively. The electromagnetic force is however modified to $\mathrm{J} \times \mathrm{B}$ since the electric field $\rho_{e} \mathrm{E}$ is assumed to be negligible. For a two dimensional flow the flow component in the $\mathrm{x}$ direction becomes

$$
\rho\left[\frac{\partial u}{\partial t}+u \frac{\partial u}{\partial x}+\mathrm{v} \frac{\partial u}{\partial y}\right]=-\frac{\partial p}{\partial x}+\mu\left[\frac{\partial^{2} u}{\partial x^{2}}+\frac{\partial^{2} u}{\partial y^{2}}\right]+\mathrm{J} \times \mathrm{B}+\rho \mathrm{g}
$$

Since the parallel plates are infinite in extend, the velocity profiles at various $\mathrm{x}$ - positions depend on $\mathrm{y}$-coordinate and not $\mathrm{x}$, however pressure in this flow is a function of $\mathrm{x}$. Further since there is no flow in the $y$ direction $v=0$. This equation thus becomes

$$
\rho\left[\frac{\partial u}{\partial t}\right]=-\frac{\partial p}{\partial x}+\mu \frac{\partial^{2} u}{\partial y^{2}}+\mathrm{J} \times \mathrm{B}+\rho \mathrm{g}
$$

In parallel flows the effect of the force of gravity is insignificant and therefore it can be ignored. Equation (12) thus reduces to

$$
\rho\left[\frac{\partial u}{\partial t}\right]=-\frac{\partial p}{\partial x}+\mu \frac{\partial^{2} u}{\partial y^{2}}+\mathrm{J} \times \mathrm{B}
$$

And for pressure gradient equals to a constant this equation simplifies to

$$
\rho\left[\frac{\partial u}{\partial t}\right]=-\mathrm{P}+\mu \frac{\partial^{2} u}{\partial y^{2}}+\mathrm{J} \times \mathrm{B}
$$

Using Reynolds decomposition on this equation yields

$$
\rho\left[\frac{\partial(\bar{u}+\dot{u})}{\partial t}\right]=-\mathrm{P}+\mu \frac{\partial^{2}(\bar{u}+\dot{u})}{\partial y^{2}}+\mathrm{J} \times \mathrm{B}
$$

Or

$$
\rho\left[\frac{\partial \bar{u}}{\partial t}+\frac{\partial \dot{u}}{\partial t}\right]=-\mathrm{P}+\mu \frac{\partial^{2} \bar{u}}{\partial y^{2}}+\mu \frac{\partial^{2} \dot{u}}{\partial y^{2}}+\mathrm{J} \times \mathrm{B}
$$

On averaging and applying the laws of ensemble equation (14) becomes

$$
\rho\left[\frac{\partial \bar{u}}{\partial t}\right]=-\mathrm{P}+\mu \frac{\partial^{2} \bar{u}}{\partial y^{2}}+\overline{\mathrm{J} \times \mathrm{B}}
$$

Now equation (17) defines Ohm's law i.e.

$$
J=\sigma(E+q \times B) \rho_{e} q
$$

And since $\rho_{\mathrm{e}} \mathrm{q}$ the displacement current is usually negligible at fluid velocity $\mathrm{q}$, the law reduces to

$$
\mathrm{J}=0
$$

Thus on substituting (18) in (17) yields

$$
\rho\left[\frac{\partial \bar{u}}{\partial t}\right]=-\mathrm{P}+\mu \frac{\partial^{2} \bar{u}}{\partial y^{2}}
$$

Conservation of Energy

This equation is the mathematical statement of the principle of conservation of energy. It states that energy cannot be created nor destroyed under normal conditions but 
can be transformed from one form to another. It is given by

$$
\rho c_{p} \frac{\mathrm{DT}}{\mathrm{Dt}}=k \nabla^{2} \mathrm{~T}+\mu \emptyset
$$

Where

$\mathrm{Cp}$ is specific heat -the amount of heat energy required to change temperature of a body by only one unit

$\mathrm{K}$ is the coefficient of thermal conductivity which depends on type of fluid.

$\frac{\mathrm{D} T}{\mathrm{D} t}$ Is the material derivative expressed as follows

$$
\frac{\mathrm{D} T}{\mathrm{D} t}=\frac{\partial T}{\partial t}+q \nabla T
$$

Or

$$
\frac{\mathrm{D} T}{\mathrm{D} t}=\frac{\partial T}{\partial t}+(\mathrm{ui}+\mathrm{vj}+\mathrm{wk}) \cdot\left(i \frac{\partial}{\partial x}+j \frac{\partial}{\partial y}+k \frac{\partial}{\partial z}\right) T
$$

Or

$$
\frac{\mathrm{D} T}{\mathrm{D} t}=\frac{\partial T}{\partial t}+\left(u \frac{\partial T}{\partial x}+\mathrm{v} \frac{\partial T}{\partial y}+w \frac{\partial T}{\partial z}\right)
$$

$k \nabla^{2} \mathrm{~T}$ This term is due to conduction of heat energy

$\emptyset$ Is the dissipative heat, which can be expressed as follows

$$
\begin{gathered}
\emptyset=\left[2\left(\left(\frac{\partial u}{\partial x}\right)^{2}+\left(\frac{\partial \mathrm{v}}{\partial y}\right)^{2}+\left(\frac{\partial w}{\partial z}\right)^{2}\right)+\left(\frac{\partial u}{\partial y}+\frac{\partial \mathrm{v}}{\partial x}\right)^{2}+\right. \\
\left.\left(\frac{\partial \mathrm{v}}{\partial z}+\frac{\partial w}{\partial y}\right)^{2}+\left(\frac{\partial u}{\partial z}+\frac{\partial w}{\partial x}\right)^{2}\right]
\end{gathered}
$$

In two dimensions equation (20) becomes

$$
\begin{aligned}
& \rho c_{p}\left[\frac{\partial T}{\partial t}+\left(u \frac{\partial T}{\partial x}+\mathrm{v} \frac{\partial T}{\partial y}\right)\right]=k \frac{\partial^{2} T}{\partial x^{2}}+k \frac{\partial^{2} T}{\partial y^{2}}+\mu\left[2 \left(\left(\frac{\partial u}{\partial x}\right)^{2}+\right.\right. \\
&\left.\left.\left(\frac{\partial \mathrm{v}}{\partial y}\right)^{2}\right)+\left(\frac{\partial u}{\partial y}+\frac{\partial \mathrm{v}}{\partial x}\right)^{2}\right]
\end{aligned}
$$

For parallel flow with the plates infinite in extend, no flow variable is a function of $\mathrm{x}$, and $\mathrm{v}=0$ (since there is no flow in the $y$ direction) equation (25) becomes

$$
\rho c_{p}\left[\frac{\partial T}{\partial t}\right]=k \frac{\partial^{2} T}{\partial y^{2}}+\mu\left[\left(\frac{\partial u}{\partial y}\right)^{2}\right]
$$

Using Reynolds decomposition on (26) yields

$$
\rho c_{p}\left[\frac{\partial T}{\partial t}+\right]=k \frac{\partial^{2} T}{\partial y^{2}}+\mu\left[\left(\frac{\partial(\bar{u}+\dot{u})}{\partial y}\right)^{2}\right]
$$

On averaging equation (27) becomes

$$
\rho c_{p}\left[\frac{\overline{\partial T}}{\partial t}\right]=k \frac{\overline{\partial^{2} T}}{\partial y^{2}}+\mu\left[+\overline{\left(\frac{\partial \overline{(u}+u)}{\partial y}\right)^{2}}\right]
$$

Or

$$
\rho c_{p}\left[\frac{\partial \bar{T}}{\partial t}\right]=k \frac{\partial^{2} \bar{T}}{\partial y^{2}}+\mu\left[\left(\frac{\partial \bar{u}}{\partial y}\right)^{2}\right]
$$

The final set of equations is thus given by

$$
\rho\left[\frac{\partial \bar{u}}{\partial t}\right]=-\mathrm{P}+\mu \frac{\partial^{2} \bar{u}}{\partial y^{2}}
$$

and

$$
\rho c_{p}\left[\frac{\partial \bar{T}}{\partial t}\right]=k \frac{\partial^{2} \bar{T}}{\partial y^{2}}+\mu\left[\left(\frac{\partial \bar{u}}{\partial y}\right)^{2}\right]
$$

\subsection{Boundary Conditions}

The initial and boundary conditions for this particular flow are

Initial conditions

$$
\mathrm{U}(0,0)=0 \quad \mathrm{~T}(0,0)=0
$$

Boundary conditions

$$
\begin{array}{cr}
\mathrm{U}(0, \mathrm{y})=0 & \mathrm{~T}(0, \mathrm{y})=0 \\
\mathrm{U}(\mathrm{t}, 0)=0 & \mathrm{~T}(\mathrm{t}, 0)=0 \\
\mathrm{U}\left(\mathrm{t}, y_{\infty}\right)=-\mathrm{U} 0 \mathrm{~T}\left(\mathrm{t}, y_{\infty}\right)=\mathrm{T} 2
\end{array}
$$

\subsection{Non-dimensionalization of Equations}

We select certain characteristic quantities. The characteristic velocity will be $\mathrm{U}_{0}$. If the temperature difference $\mathrm{T}-\mathrm{T}_{2}$ is divided by the maximum possible temperature difference $T_{1}-T_{2}$, a dimensionless form of the dependent variable may be defined as $\theta=\left(T-T_{2} / T_{1}-T_{2}\right)$ where $\mathrm{T}_{1}$ and $\mathrm{T}_{2}$ are temperatures next to the plate and free stream temperature and $0 \leq \theta \leq 1$

The independent and dependent variables may then be non-dimensionalised according to

$$
\begin{gathered}
\mathrm{y}=y^{*} \mathrm{~L} \\
\mathrm{u}=\mathrm{u}^{*} \mathrm{u} 0 \\
\mathrm{t}=\frac{t^{*} L}{U_{0}} \mathrm{p}=\mathrm{p} 0 \mathrm{p}^{*} \\
\left(T-T_{2}\right)=\left(T_{1}-T_{2}\right) \theta
\end{gathered}
$$

Equation (30) thus becomes

$$
\rho\left[\frac{u_{0}^{2} \partial \overline{u^{*}}}{L \partial t^{*}}\right]=-\mathrm{P}_{0} \mathrm{p}^{*}+\frac{\mu \mathrm{u}_{0}}{L^{2}} \frac{\partial^{2} \overline{u^{*}}}{\partial y^{* 2}}
$$

Simplifying and dropping the asterisks yields

$$
\frac{u_{0}^{2} \partial \bar{u}}{L \partial t}=-\frac{1}{\rho} \mathrm{P}_{0} \mathrm{p}+\frac{\mathrm{vu}_{0}}{L^{2}} \frac{\partial^{2} \bar{u}}{\partial y^{2}}
$$

Dividing by $\frac{u_{0}^{2}}{L}$ yields

$$
\frac{\partial \bar{u}}{\partial t}=-\frac{\mathrm{LP}_{0} \mathrm{p}}{\rho \mathrm{u}_{0}^{2}}+\frac{v}{\mathrm{u}_{0} L} \frac{\partial^{2} \bar{u}}{\partial y^{2}}
$$

Similarly equation (31) can be rearranged as follows

$$
\frac{\partial \bar{T}}{\partial t}=\frac{k}{\rho c_{p}} \frac{\partial^{2} \bar{T}}{\partial y^{2}}+\frac{\mu}{c_{p}}\left(\frac{\partial \bar{u}}{\partial y}\right)^{2}
$$

And on substituting (32) in (36) yields

$$
\frac{\mathrm{u}_{0}}{L}\left(T_{1}-T_{2}\right) \frac{\partial \bar{\theta}}{\partial t^{*}}=\frac{k}{\rho c_{p} L}\left(T_{1}-T_{2}\right) \frac{\partial^{2} \bar{\theta}}{\partial y^{* 2}}+\frac{\mu}{\rho c_{p}}\left(\frac{\mathrm{u}_{0}}{L} \frac{\partial \overline{u^{*}}}{\partial y^{*}}\right)^{2}
$$


On simplifying, dropping the asterisks and dividing by $\frac{\mathrm{u}_{0}}{L}\left(T_{1}-T_{2}\right)$ yields

$$
\frac{\partial \bar{\theta}}{\partial t}=\frac{k}{\rho L c_{p} \mathrm{u}_{0}} \frac{\partial^{2} \bar{\theta}}{\partial y^{2}}+\frac{v}{\mathrm{u}_{0} L c_{p}} \frac{u_{0}^{2}}{\left(T_{1}-T_{2}\right)}\left(\frac{\partial \bar{u}}{\partial y}\right)^{2}
$$

or

$$
\begin{gathered}
\frac{\partial \bar{u}}{\partial t}=-P_{c}+\frac{1}{R_{e}} \frac{\partial^{2} \bar{u}}{\partial y^{2}} \\
\frac{\partial \bar{\theta}}{\partial t}=\frac{1}{P_{e}} \frac{\partial^{2} \bar{\theta}}{\partial y^{2}}+\frac{1}{R_{e}} E_{c}\left(\frac{\partial \bar{u}}{\partial y}\right)^{2}
\end{gathered}
$$

On non-dimensionalisation of the initial and boundary conditions and dropping the asterisks we get

$$
\begin{gathered}
\mathrm{U}(0,0)=0 \theta(0,0)=0 \\
\mathrm{U}(0, \mathrm{y})=0 \quad \theta(0, \mathrm{y})=0 \\
\mathrm{U}(\mathrm{t}, 0)=0 \mathrm{U}\left(\mathrm{t}, y_{\infty}\right)=-1 \\
\theta(\mathrm{t}, 0)=0 \theta\left(\mathrm{t}, y_{\infty}\right)=1
\end{gathered}
$$

\subsection{Important Non-Dimensional Numbers}

From equations (35) and (38) the following nondimensional numbers have emerged.

\subsubsection{Hydrodynamic Reynolds Number $\boldsymbol{R}_{e}$}

This is the ratio of inertia force to viscous force. It gives the relative significance of inertia force in fluid flow problem and is expressed as

$$
R_{e}=\frac{\rho U_{0}}{\mu}=\frac{U_{0} L}{\mu}
$$

When Reynolds number is large, the inertia forces are predominant and the effects of viscosity are negligible and when its value is small, the flow is dominated by viscous force and inertia force can be neglected.

\subsubsection{Peclet Number $P_{e}$}

This is a non-dimensional number $\mathrm{P}_{\mathrm{e}}$ defined as

$$
P_{e}=\frac{\rho U L C_{p}}{K}
$$

This number is small when viscous force is small while thermal force is large.

\subsubsection{The Eckert Number $E_{c}$}

It is the ratio of kinetic energy of the flow to the thermal energy.

It is expressed as

$$
E_{c}=\frac{U_{0}^{2}}{C_{p} \Delta T}
$$

\subsubsection{Pressure Number}

This is a non-dimensional number $P_{c}$ defined as

$$
P_{c}=\frac{P}{\rho U^{2}}
$$

Or

$$
P_{c}=\frac{P L^{2}}{\rho U^{2} L^{2}}
$$

This is the ratio of pressure forces to the inertia force.

\section{Method of Solution}

\subsection{Finite Difference Method}

In this study the equations governing fluid flow are nonlinear and thus their exact solution is not possible; in order to solve these equations a fast and stable difference method has been developed. The difference method used should be consistent, stable and convergent. A method is convergent if as more grid points are taken or step size decreased, the numerical solution converges to the exact solution. A method is consistent if the truncation error tends to zero as the step size decreases, and stable if the effect of any single fixed round off error is bounded.

We shall now apply a numerical method approach to solve the inhomogeneous partial differential equations. The particular method for this problem will be the central finite difference method, with a uniform time step (m) and spatial step $(n)$. It is defined by expanding $f\left(x_{0}+h\right)$ and $f\left(x_{0}-h\right)$ using the Taylors series and then solving the two;

$$
\begin{gathered}
f(x+h)=f(x)+h f^{I}(x)+\frac{h^{2}}{2} f^{I I}(x)+\frac{h^{3}}{3} f^{I I I}(x) \\
f(x-h)=f(x)-h f^{I}(x)+\frac{h^{2}}{2} f^{I I}(x)-\frac{h^{3}}{3} f^{I I I}(x)
\end{gathered}
$$

By subtracting (44) from (43) and for sufficiently small, h yields.

$$
f^{I I}(x)=\frac{f(x-h)-2 f(x)+f(x+h)}{h^{2}}
$$

Similarly by adding (44) to (43) and for sufficiently small, $\mathrm{h}$ yields

$$
f^{I}(x)=\frac{f(x+h)-f(x-h)}{2 \mathrm{~h}}
$$

In two dimensions the following partial derivatives can be expressed as follows

$$
\begin{gathered}
\frac{\partial^{2} \bar{u}}{\partial y^{2}} u^{I I}=\frac{u_{i, j+1}-2 u_{i, j}+u_{i, j-1}}{\mathrm{n}^{2}} \\
\frac{\partial^{2} \bar{\theta}}{\partial y^{2}} \theta^{I I}=\frac{\theta_{i, j+1}-2 \theta_{i, j}+\theta_{i, j-1}}{\mathrm{n}^{2}} \\
\frac{\partial \bar{u}}{\partial y} u^{I}=\frac{u_{i, j+1}-u_{i, j-1}}{2 \mathrm{n}} \\
\frac{\partial \theta}{\partial y} \theta^{I}=\frac{\theta_{i, j+1}-\theta_{i, j-1}}{2 \mathrm{n}}
\end{gathered}
$$

Let the mesh point at time $t$ be denoted by $f^{I}(x)$. Then the forward difference for the first order derivative with respect to time $\mathrm{t}$ will be. 


$$
f^{I}(x)=\frac{f(x+h)-f(x)}{\Delta \mathrm{t}}
$$

And thus

$$
\begin{aligned}
& \frac{\partial u}{\partial t} u^{I}=\frac{u_{i+1, j}-u_{i, j}}{\mathrm{~m}} \\
& \frac{\partial \theta}{\partial t} \theta^{I}=\frac{\theta_{i+1, j}-\theta_{i, j}}{\mathrm{~m}}
\end{aligned}
$$

Equations (39) and (40) thus becomes

$$
\frac{\partial \bar{u}}{\partial t}=-P_{c}+\frac{1}{R_{e}} \frac{\partial^{2} \bar{u}}{\partial y^{2}}
$$

Substituting (central difference method)

$$
\begin{aligned}
\frac{\partial u}{\partial t} u^{I}=\frac{u_{i+1, j}-u_{i, j}}{\mathrm{~m}} & \\
\frac{\partial \theta}{\partial t} \theta^{I}=\frac{\theta_{i+1, j}-\theta_{i, j}}{\mathrm{~m}} \frac{\partial^{2} \bar{\theta}}{\partial y^{2}} \theta^{I I} & =\frac{\theta_{i, j+1}-2 \theta_{i, j}+\theta_{i, j-1}}{\mathrm{n}^{2}} \frac{\partial \bar{u}}{\partial y} u^{I}=\frac{u_{i, j+1}-u_{i, j-1}}{2 \mathrm{n}} \\
\frac{\theta_{i+1, j}-\theta_{i, j}}{\mathrm{~m}} & =\frac{1}{P_{e}}\left(\frac{\theta_{i, j+1}-2 \theta_{i, j}+\theta_{i, j-1}}{\mathrm{n}^{2}}\right)+\frac{1}{R_{e}} E_{c}\left(\frac{u_{i, j+1}-u_{i, j-1}}{2 \mathrm{n}}\right)^{2} \\
\theta_{i+1, j}- & \theta_{i, j}=\frac{\mathrm{m}}{P_{e}}\left(\frac{\theta_{i, j+1}-2 \theta_{i, j}+\theta_{i, j-1}}{\mathrm{n}^{2}}\right)+\frac{\mathrm{m} E_{c}}{R_{e}}\left(\frac{u_{i, j+1}-u_{i, j-1}}{2 \mathrm{n}}\right)^{2} \\
\theta_{i+1, j} & =\theta_{i, j}+\frac{\mathrm{m}}{P_{e}}\left(\frac{\theta_{i, j+1}-2 \theta_{i, j}+\theta_{i, j-1}}{\mathrm{n}^{2}}\right)+\frac{\mathrm{m} E_{c}}{R_{e}}\left(\frac{u_{i, j+1}-u_{i, j-1}}{2 \mathrm{n}}\right)^{2}
\end{aligned}
$$

The two final equations to be solved are thus

$$
\begin{gathered}
u_{i+1, j}=u_{i, j}-\mathrm{m} P_{c}+\frac{\mathrm{m}}{R_{e}}\left(\frac{u_{i, j+1}-2 u_{i, j}+u_{i, j-1}}{\mathrm{n}^{2}}\right) \\
\theta_{i+1, j}=\theta_{i, j}+\frac{\mathrm{m}}{P_{e}}\left(\frac{\theta_{i, j+1}-2 \theta_{i, j}+\theta_{i, j-1}}{\mathrm{n}^{2}}\right)+\frac{\mathrm{m} E_{c}}{R_{e}}\left(\frac{u_{i, j+1}-u_{i, j-1}}{2 \mathrm{n}}\right)^{2}
\end{gathered}
$$

\subsection{Definition of Mesh}

In order to give a relation between the partial derivatives in the differential equation and the function values at the adjacent nodal points, we use a uniform mesh. Let the t-y plane be divided into a network of uniform rectangular cells of width $m$ and height $n$ as shown in figure 3 below.

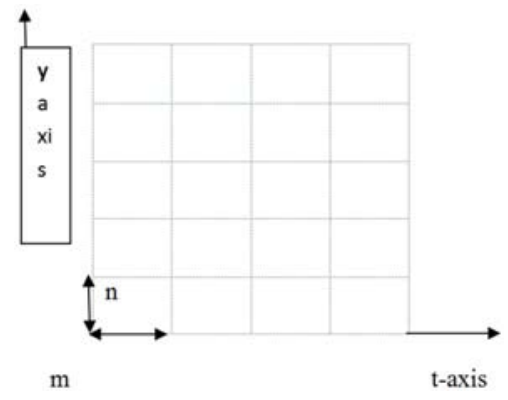

Fig. 3. Uniform rectangular mesh.

Since $\mathrm{t}$-axis is along the infinite horizontal plate then $\mathrm{t}$ varies from 0 to infinity. If we set $i=21$ to correspond to $t=\infty$ and $m=n=0.1$ then we have

$$
t=i m=21 \times 0.1=2.1
$$

Initial and boundary conditions

$$
\begin{gathered}
\mathrm{U}(0,0)=0 \theta(0,0)=0 \\
\mathrm{U}(0,2.1)=0 \theta(0,2.1)=0 \\
\mathrm{U}(2.1,0)=0 \mathrm{U}(2.1,2.1)=-1 \\
\theta(2.1,0)=0 \theta(2.1,2.1)=1
\end{gathered}
$$

The computations are done when $\mathrm{m}=\mathrm{n}=0.1$

Reynolds Number $\mathrm{Re}=10$

Pressure Coefficient $\mathrm{Pc}=0.8$

Pecklet Number is 1

Eckert Number varies from 0 to 0.35 to 0.7

\section{Results and Discussions}

\subsection{Tabular and Graphical Results}

Table 1. The effect of the Eckert number on velocity.

\begin{tabular}{llll}
\hline & $\mathbf{E c}=\mathbf{0 . 1}$ & $\mathbf{E c}=\mathbf{0 . 3 5}$ & $\mathbf{E c}=\mathbf{0 . 7}$ \\
\hline 0 & 0 & 0 & 0 \\
1 & 0.42523 & 1.3543 & 2.01921 \\
\hline
\end{tabular}




\begin{tabular}{llll}
\hline & $\mathbf{E c}=\mathbf{0 . 1}$ & $\mathbf{E c}=\mathbf{0 . 3 5}$ & $\mathbf{E c}=\mathbf{0 . 7}$ \\
\hline 2 & 0.78487 & 2.08859 & 2.75745 \\
3 & 1.04315 & 2.50426 & 3.14721 \\
4 & 1.27043 & 2.73271 & 3.36456 \\
5 & 1.45 & 2.90498 & 3.49561 \\
6 & 1.56888 & 2.92693 & 3.57458 \\
7 & 1.61894 & 2.81563 & 3.61645 \\
8 & 1.59756 & 2.59634 & 3.54562 \\
9 & 1.5079 & 2.29903 & 3.36364 \\
10 & 1.35856 & 1.97878 & 3.11153 \\
11 & 1.16292 & 1.59378 & 2.683244 \\
\hline
\end{tabular}

velocity profiles

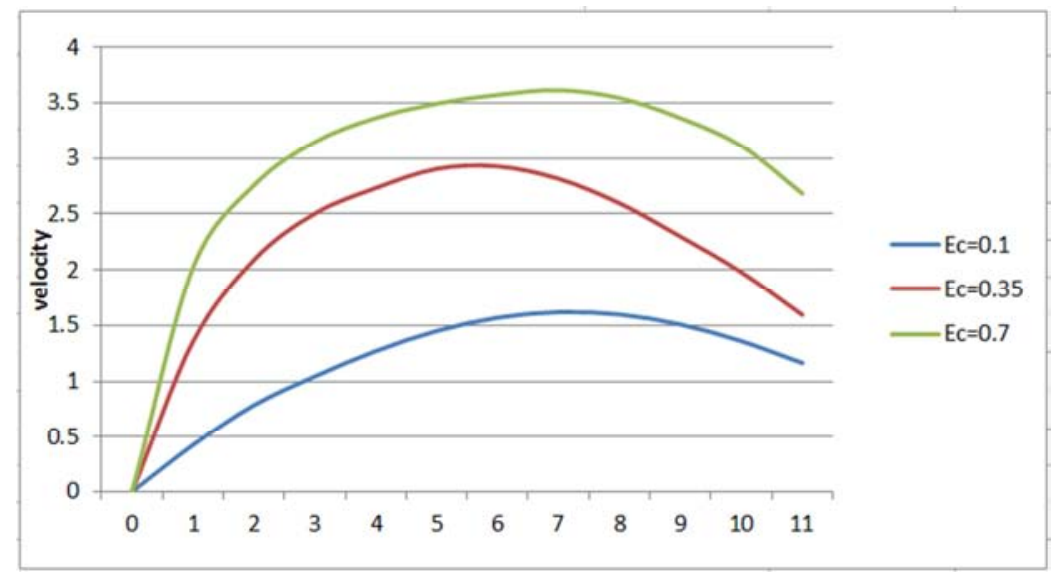

Fig. 4. Velocity increases with an increase in Eckert number.

Table 2. The effect of the Eckert number on temperature.

\begin{tabular}{llll}
\hline & $\mathbf{E} \mathbf{c}=\mathbf{0 . 1}$ & $\mathbf{E c}=\mathbf{0 . 3 5}$ & $\mathbf{E c}=\mathbf{0 . 7}$ \\
\hline 1 & 0.075268 & 0.039551 & 0.01134 \\
2 & 0.185963 & 0.115195 & 0.05502 \\
3 & 0.297149 & 0.214877 & 0.127943 \\
4 & 0.430054 & 0.324874 & 0.22256 \\
5 & 0.558765 & 0.434165 & 0.319759 \\
6 & 0.671191 & 0.551459 & 0.400204 \\
7 & 0.776117 & 0.658201 & 0.471402 \\
8 & 0.848983 & 0.747214 & 0.528128 \\
9 & 0.913048 & 0.824583 & 0.584311 \\
10 & 0.963912 & 0.877218 & 0.631193 \\
11 & 0.992123 & 0.911016 & 0.67073 \\
\hline
\end{tabular}

Temperature Profile

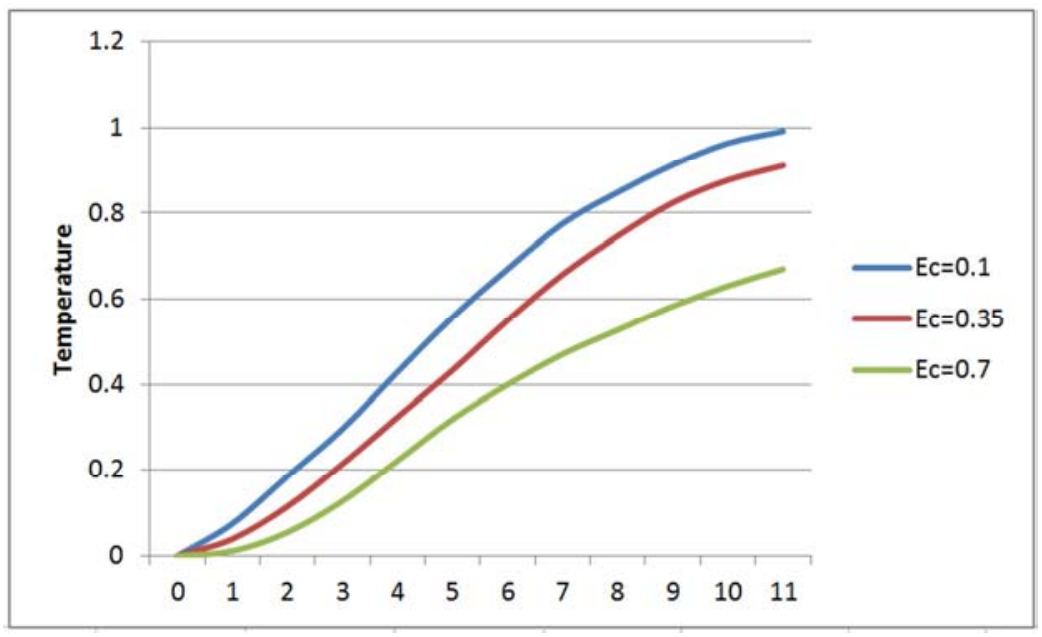

Fig. 5. Temperature decreases with an increase in Eckert number. 


\subsection{Discussion of the Results}

The effects of the Eckert number on the temperature and velocity profiles.

ii. An increase in the Eckert number causes an increase in the velocity (figure 4).

iii. The graph of velocity shows that velocity increases up to a maximum value i.e. the free stream velocity, and then declines to the velocity at which the upper plate is moving.

iv. An increase in the Eckert number causes a decrease in the temperature (figure 5).

v. The graph of temperature shows that temperature increases from zero; as the lower plate is maintained at zero temperature, and increases towards the temperature at which the upper plate is maintained.

\subsection{Conclusion}

A model for the problem was developed carefully putting into considerations appropriate assumptions. A finite difference method was used to solve the resulting equations. From the results it is evident that Eckert number has a fundamental influence in the dynamics of fluid flow and in particular temperature and velocity. It has been shown that an increase in the Eckert number causes an increase in the velocity. Different values of the number were investigated and found to increase velocity as the numbers increased. Velocity however increases up to the free stream velocity, and then declines to the velocity at which the upper plate is moving. It is also evident that an increase in the Eckert number causes a decrease in the temperature. However temperature increases from zero as the lower plate is maintained at zero temperature, and increases in conformity with the boundary conditions.

\section{Nomenclature}

\begin{tabular}{|c|c|}
\hline Symbols & Quantities \\
\hline $\mathrm{B}$ & Magnetic field vector $\left(\mathrm{wbm}^{-2}\right)$ \\
\hline $\mathrm{B}_{\mathrm{X}}, \mathrm{B}_{\mathrm{Y}, \mathrm{B}_{\mathrm{Z}}}$ & Magnetic flux in $\mathrm{x}, \mathrm{y}, \mathrm{z}$ directions respectively $\left(\mathrm{wbm}^{-2}\right)$ \\
\hline $\mathrm{C}_{\mathrm{P}}$ & Specific heat at constant pressure $\left(\mathrm{KJ} / \mathrm{Kg}^{0} \mathrm{C}\right)$ \\
\hline $\mathrm{D}$ & Electric displacement vector $\left(\mathrm{Cm}^{-2}\right)$ \\
\hline$\frac{D q}{D}$ & Material derivative of velocity \\
\hline $\begin{array}{l}D t \\
\mathrm{e}\end{array}$ & Unit electric charge $(\mathrm{C})$ \\
\hline E & Electric field intensity vector $\left(\mathrm{vm}^{-1}\right)$ with components $\mathrm{E}_{\mathrm{X}, \mathrm{E}} \mathrm{E}_{\mathrm{Y}} \mathrm{E}_{\mathrm{Z}}$ \\
\hline $\mathrm{F}$ & Body force $(\mathrm{N})$ \\
\hline $\mathrm{H}$ & Magnetic field strength with components $\mathrm{H}_{\mathrm{X}}, \mathrm{H}_{\mathrm{Y}} \mathrm{H}_{\mathrm{Z}}$ \\
\hline $\mathrm{i}, \mathrm{j}, \mathrm{k}$ & Unit vectors in the $\mathrm{x}, \mathrm{y}, \mathrm{z}$ directions respectively \\
\hline J & Current density vector $\left(\mathrm{Am}^{-2}\right)$ with components $\mathrm{J}_{X}, \mathrm{~J}_{Y}, \mathrm{~J}_{Z}$ \\
\hline K & Thermal Conductivity $\left(\mathrm{wm}^{-1} \mathrm{k}^{-1}\right)$ \\
\hline $\mathrm{L}$ & Characteristic length $(\mathrm{m})$ \\
\hline $\mathrm{P}$ & Pressure force vector $\left(\mathrm{Nm}^{-2}\right)$ \\
\hline \multicolumn{2}{|c|}{ respectively } \\
\hline $\mathrm{P}_{\mathrm{r}}$ & Prandtl number $(\mathrm{Cp} \mu / \mathrm{k})$ \\
\hline $\operatorname{Re}$ & Hydrodynamics Reynolds number $\frac{\text { UoL }}{V}$ \\
\hline $\mathrm{R} \sigma$ & Magnetic Reynolds number $\sigma \mu_{\mathrm{e}} \mathrm{U}_{\mathrm{OL}}$ \\
\hline $\mathrm{R}_{\mathrm{h}}$ & Magnetic pressure number $\frac{B_{0}{ }^{2}}{\rho \mu_{e} U_{0}{ }^{2}}$ \\
\hline $\mathrm{t}$ & Time (s) where $\mathrm{t}=\frac{L t^{*}}{U_{0}}$ \\
\hline$t^{*}$ & Dimensionless time \\
\hline $\mathrm{T}$ & General fluid temperature $(\mathrm{K})$ \\
\hline $\bar{U}$ & The steady component of $U$ \\
\hline \multicolumn{2}{|c|}{$u^{\prime}$ The fluctuating part of $U$} \\
\hline $\mathrm{U}_{0}$ & Characteristic velocity $\left(\mathrm{ms}^{-1}\right)$ \\
\hline $\mathrm{U}^{*}$ & Non-dimensional velocity \\
\hline \multicolumn{2}{|c|}{$\mathrm{u}^{*}, \mathrm{v}^{*}, \mathrm{w}^{*}$ Dimensionless velocity components } \\
\hline \multicolumn{2}{|c|}{$\mathrm{x}^{*}, \mathrm{y}^{*}, \mathrm{z}^{*}$ Dimensionless Cartesian co-ordinates } \\
\hline$\rho$ & Fluid density $\left(\mathrm{kgm}^{-3}\right)$ \\
\hline$\rho_{\mathrm{e}}$ & Electric charge density $\left(\mathrm{cm}^{-2}\right)$ \\
\hline
\end{tabular}




\begin{tabular}{ll}
\hline$\mu$ & Coefficient of viscosity $\left(\mathrm{kgm}^{-1} \mathrm{~s}^{-1}\right)$ \\
$\mu_{\mathrm{e}}$ & Magnetic permeability $\left(\mathrm{Hm}^{-1}\right)$ \\
$\sigma$ & Electric conductivity $\left(\Omega^{-1} \mathrm{~m}^{-1}\right)$ \\
$\nabla$ Gradient operator $\left(i \frac{d}{d x}+j \frac{d}{d y}+k \frac{d}{d z}\right)$ & \\
$\nabla^{2}$ & Laplacian operator $\left(\frac{d^{2}}{d x^{2}}+\frac{d^{2}}{d y^{2}}+\frac{d^{2}}{d z^{2}}\right)$ \\
$\Phi$ & Viscous dissipation function $\left(\mathrm{s}^{-2}\right)$ \\
$\theta$ & Dimensionless fluid temperature \\
\hline Acronyms and Abbreviations & \\
\hline MHD & Magnetohydrodynamics \\
LHD & Left hand side \\
RHD & Right hand side \\
RD & Reynolds Decomposition \\
\hline
\end{tabular}

\section{References}

[1] Ahmed, M. S. El-Aziz, M. A. Abo-Eldahab, E. M. AbdElfatah, I. (1992). "Effect of variable density on hydromagnetic mixed convection flow of a non Newtonian fliud past past a moving vertical plate" Communications in Nonlinear Science and Numerical Simulation, Volume 14, Issue 5, Pages 2202-2214.

[2] Bhaskara, S \& Bathaiah, V. (1980). "MHD flow of a viscous incompressible and slightly conducting fluid between a parallel flat wall and a wavy wall" International Journal of Heat and Mass Transfer, Volume 32, Issues 13-14, Pages 1390-1395.

[3] Betil, F. D. (2007). "Magneto hydrodynamics" Scholarpedia 2(4): 2295 pp1-5.

[4] Calvert, J. B. (2002). "Magnetohydrodynamics" New York: Inter science.

[5] Chandra, S. V. (2005) "MHD flow of an electrically conducting fluid between two parallel infinite plates when the upper plate is made to move with constant velocity while the lower plate is stationary" International Journal of Heat and Mass Transfer, Volume 52, Issues 13-14, Pages 3390-3395.

[6] Chaturvedi, N. (1996). "MHD flow past an infinite porous plate with variable suction" Energy Conversion and Management, Volume 37, Issue 5, P p 623-627.

[7] Cowling, T. G. (1957). "Magnetohydrodynamics," New York: Interscience.

[8] Denis, R. (1980). Encyclopedia of agricultural, food, and biological engineering pp 560-568.

[9] Faraday, M. (1831). "Experimental Researches in Chemistry and Physics". London: Richard Taylor and William Francis. pp. 33-53.

[10] Gupta, V., \& Gupta, S. K. (1991). "Fluid mechanics and its applications," Wiley Eastern Limited, New Delhi, India pp 100-102

[11] Hartman, J, \& Lazarus, F. (1937). "Experimental investigations on the flow of mercury in a homogeneous magnetic field" pp 1-5.

[12] Kalyuit, M. N. (1986). "Development of the flow field of an electrically conducting fluid in an inhomogeneous magnetic field" Reed Educational and Professional Publishing Ltd, pp29-35.

[13] Kinyanjui, M., Chaturvedi, N., \& Uppal, S. M. (1998). “MHD stokes problem for a vertical infinite plate in a dissipative rotating fluid with a hall current" Energy Conversion and Management, Volume 39, Issues 5-6, Pages 541-548

[14] Kinyanjui, M, Kwanza, J. K., \& Uppal S. M. (2001). "Magnetohydrodynamic free convection heat and mass transfer of a heat generating fluid past an impulsively started infinite vertical porous plate with Hall current and radiation absorption" Energy Conversion and Management, Volume 42, Issue 8, Pages 917-931.

[15] Kumar, A. S., Singh, N. P., Singh, U., \& Singh, H. (2009). "Convective flow past an accelerated porous plate in rotating system in presence of magnetic field" International Journal of Heat and Mass Transfer, Volume 52, Issues 13-14, Pages 3390-3395.

[16] Jackson, J. D. (1975). "Classical Electrodynamics," second edition $\mathrm{pp}$ 1-5.

[17] Landau, L. D., \& Lifshitz, E. M. (1982). "Fluid Mechanics," Reed Educational and Professional Publishing Ltd, pp 129135 .

[18] Molokov, S. Y., \& Allen, J. E. (1992). J. phys. D: Appl. phys.25, pp 395-400.

[19] Plumpton, F. (1961). "An introduction to Magnetofluid Mechanics" Oxford University Press.

[20] Rossow, V. J. (1958): NASA Report No.1358.

[21] Samiulhaq, Khan I, Ali F, Shafie S (2012). "MHD free convection flow in a porous medium with thermal diffusion and ramped wall temperature". J Phys Soc Jpn 81: 4401.

[22] Stewartson, K. (1951): Quart. J. Mcch. Appl. Math. 4,182.

[23] Stokes, G. C. (1951). Cambr. Phil. Trans 9, 8.

[24] Walker, J. S. (1971). "Liquid metal flow through a thin walled elbow in a plane perpendicular to a uniform magnetic field" International Journal of Engineering Science, Volume 24, Issue 11, Pages 1741-1754. 